Lie Theory and Its Applications in Physics VII

eds. H.-D. Doebner and V.K. Dobrev, Heron Press, Sofia, 2008

\title{
Monodromy analysis of the computational power of the Ising topological quantum computer
}

\author{
Andre Ahlbrecht ${ }^{2,3}$, Lachezar S. Georgiev ${ }^{1,2}$ and \\ Reinhard F. Werner ${ }^{2,3}$ \\ ${ }^{1}$ Institute for Nuclear Research and Nuclear Energy, \\ Bulgarian Academy of Sciences, \\ 72 Tzarigradsko Chaussee, 1784 Sofia, Bulgaria \\ ${ }^{2}$ Insitut für Mathematische Physik, Technische Universität Braunschweig, \\ Mendelssohnstr. 3, 38106 Braunschweig, Germany \\ ${ }^{3}$ Insitut für Theoretische Physik, Leibniz Universität Hannover, Appelstr. 2, \\ 30167 Hannover, Germany
}

\begin{abstract}
We show that all quantum gates which could be implemented by braiding of Ising anyons in the Ising topological quantum computer preserve the $n$ qubit Pauli group. Analyzing the structure of the Pauli group's centralizer, also known as the Clifford group, for $n \geq 3$ qubits, we prove that the image of the braid group is a non-trivial subgroup of the Clifford group and therefore not all Clifford gates could be implemented by braiding. We show explicitly the Clifford gates which cannot be realized by braiding estimating in this way the ultimate computational power of the Ising topological quantum computer.
\end{abstract}

\section{Introduction}

Quantum computers are expected to be much more powerful than the classical supercomputers due to a combination of quantum phenomena such as coherent superpositions, entanglement and paralelism [1]. Topological Quantum Computers are a class of quantum computers in which information is encoded in non-local topological quantum numbers, such as the anyon fusion channels, and quantum gates are implemented by anyon braiding protecting in this way quantum information processing from noise [2,3].

In this paper we will analyze the properties of the monodromy subgroup of the braid group for the Ising anyon TQC and will demonstrate that all quantum 
gates which can be executed by braiding are Clifford gates [1,4], that are important for fault-tolerant quantum computation, while not all Clifford gates could be implemented by braiding Ising anyons.

\subsection{What are anyons?}

Anyons are particle-like collective excitations, which are supposed to exist in strongly correlated two-dimensional electron systems, that may carry fractional electric charge (measured in shot-noise experiments with Laughlin quantum Hall states) and obey exotic exchange statistics: due to the properties of the twodimensional rotation group $S O(2)$ the statistics under exchange of identical particles is governed by representations of the braid group rather than the permutation group. The Abelian anyon many-body states belong to one-dimensional representations of the braid group and acquire nontrivial phases when neighboring anyons are exchanged. If the many-body states belong to a higher-dimensional representation of the braid group the corresponding particles are called nonAbelian anyons, or plektons, and the results of particle exchanges are not only phases but could also be more general unitary transformations [3,5].

Anyons might be observable in fractional quantum Hall samples as well as in high-temperature superconductors [5] and cold atoms in optical lattices (intersecting laser beams).

\subsection{Fusion paths: labeling anyonic states of matter}

The fact that the exchanges of non-Abelian anyons may generate non-trivial matrices acting on the many-body state implies that in fact this state must belong to a degenerate multiplet of states with many anyons at fixed positions. Therefore the anyon's positions and quantum numbers are not sufficient for specifying a multi-anyon state, i.e., some additional non-local information is necessary. It appears that in order to specify the state of many non-Abelian anyons we need to fix the fusion channels of any two neighbors. The reason is that the same multi-anyon configuration may correspond to different independent states (CFT blocks) because of the possibility of multiple fusion channels. Consider, the process of fusing two anyons of type " $a$ " and " $b$ ", represented by some operators $\Psi_{a}$ and $\Psi_{b}$. The result is expressed by the following fusion rule

$$
\Psi_{a} \times \Psi_{b}=\sum_{a=1}^{g} N_{a b}^{c} \Psi_{c}
$$

where $N_{a b}^{c}$ are the fusion coefficients. There are two classes of anyons:

- Abelian: $\forall a, b \exists ! c$ such that $N_{a b}^{c} \neq 0$

- non-Abelian: if for some $a$ and $b N_{a b}^{c} \neq 0$ for more than one $c$

In TQC we are interested in information encoding for non-Abelian anyons: because by definition there are more than 1 fusion channels we could encode information in the index of the fusion channel. 
For example, for Ising anyons $\Psi_{I}(z)=\sigma(z)$, represented by the chiral spin filed operator of CFT dimension $\Delta=1 / 16$, which are characterized by the fusion rule

$$
\sigma \times \sigma=\mathbb{I}+\psi,
$$

where $\psi$ is the Majorana fermion, information is encoded as follows: if a pair of $\sigma$ fields is in the vacuum fusion channel we call the state $|0\rangle$, while if it is in the Majorana channel we call the state $|1\rangle$

$$
\begin{array}{rll}
|0\rangle=(\sigma, \sigma)_{\mathbb{I}} & \longleftrightarrow & \sigma \times \sigma \rightarrow \mathbb{I} \\
|1\rangle=(\sigma, \sigma)_{\psi} & \longleftrightarrow & \sigma \times \sigma \rightarrow \psi
\end{array}
$$

(the subscript of a pair denotes its fusion channel). The important point here is that fusion channel is independent of the fusion process details-it is a topological quantity. It is also non-local because the fusion channel is independent of the anyon separation and is preserved even for large separation of anyons. Finally, it is robust and persistent-if we fuse two particles and then split them again the fusion channel does not change. Therefore, the message is that, in addition to the positions and all local quantum numbers of the anyons, a multi-anyon state could be unambiguously specified by the fusion path, i.e., the concatenation of elementary fusion channels for each neighbors in an array of anyonic fields at fixed positions. This is conveniently presented in the form of Bratteli diagrams (see, [6] for more details).

\subsection{Quantum gates: adiabatic transport of anyons}

As we mentioned above, we intend to execute quantum gates over our quantum register by adiabatic exchange of anyons. The adiabatic approximation requires that the system has a gapped Hamiltonian, i.e., a non-zero energy gap $\Delta$ to exist between the ground state and the excitations. The external parameters describing the transport are the anyons positions $R_{1}, \ldots, R_{k}$ in the plane (pinned by trapping potentials). We recall that we are interested in operations which keep the anyon's positions fixed so that final configuration is at most a permutation of the anyons in the original one. The elementary braiding processes are realized by taking one anyon adiabatically around its neighbor on a large time scale $t \in[0, T]$ with $T \gg \Delta^{-1}$ (for example, if the energy gap of the $\nu=5 / 2 \mathrm{FQH}$ state is $\Delta=500 \mathrm{mK}$ then the minimum time interval is $T_{\min } \sim 10^{-10} \mathrm{~s}$ ). The adiabatic theorem for a non-degenerate ground state implies that if the system is initially in the ground state then the final state after executing a complete loop in the parameter space, is up to phase again the ground state

$$
\psi_{f}^{\left(R_{1}, \ldots, R_{k}\right)}\left(z_{1}, \ldots, z_{N}\right)=\mathrm{e}^{i \phi} \psi_{i}^{\left(R_{1}, \ldots, R_{k}\right)}\left(z_{1}, \ldots, z_{N}\right),
$$

where $z_{i}$ are the coordinates of the electrons while $R_{j}$ are the coordinates of the anyons in the plane. However, if the ground state is degenerate and separated by a gap from the excited states, then the adiabatic theorem implies that the final state after traversing a complete loop is again a ground state from the same degenerate multiplet but may be different from the original one. In this case the 
phase $\mathrm{e}^{i \phi}$ in (1) must be replaced by a unitary operator acting on the multiplet [3].

Ignoring the dynamical phase $\mathrm{e}^{i \frac{1}{\hbar} \int d t E(\mathbf{R}(t))}$ contribution to $\mathrm{e}^{i \phi}$ we will focus on the (non-) Abelian Berry phase $\mathrm{e}^{i \alpha}$ defined by

$$
\alpha=\oint d \overrightarrow{\mathbf{R}} \cdot\left\langle\psi(\overrightarrow{\mathbf{R}})\left|\vec{\nabla}_{\mathbf{R}}\right| \psi(\overrightarrow{\mathbf{R}})\right\rangle, \quad \overrightarrow{\mathbf{R}}=\left(R_{1}, \ldots, R_{k}\right)
$$

There are three contributions to the Berry phase (2), which have different status with respect to the topological protection:

- geometrical phase which is of the type of the Aharonov-Bohm phase

- topological phase (quasiparticle statistics, independent of the geometry)

- monodromy of CFT wave functions with anyons

The geometrical phase is proportional to the area of the loop and is not topologically protected. When the many-body states are described by chiral CFT wave functions which are also orthonormal, the Berry connection is trivial [7]. Because of this, the entire effect of the adiabatic transport is given by the explicit monodromy of the multi-valued CFT correlators. Therefore, when we construct quantum gates for TQC, we can directly deal with the braid generators and forget about the Berry connection induced by the adiabatic transport.

\section{$2 n$ Ising qubits: $2 n+2$ Ising anyons on antidots}

Because the dimension of the computational space, spanned by the Pfaffian wave functions with $2 n+2$ Ising anyons at fixed positions $\eta_{1}, \ldots \eta_{2 n+2}$ in the plane [8], is $2^{n}$ we could use the many-body states represented by these wave functions to realize $n$ Ising qubits. Our qubit encoding scheme is roughly that we use one pair of $\sigma$ fields to represent one qubit, whose state is $|0\rangle$ if the pair is in the fusion channel of the vacuum or $|1\rangle$ if it is in the Majorana channel. However, since we would like to represent qubits by chiral CFT correlation functions of Ising $\sigma$ fields, which are non-zero only if the total fermion parity inside the correlator is trivial, we need one extra pair of $\sigma$ fields, which is inert from the viewpoint of TQC but compensates if necessary total parity of the encoded qubits, i.e., our one-qubit states could be written as 4-pt correlators

$$
\left|c_{1}\right\rangle=\left\langle(\sigma \sigma)_{c_{1}}(\sigma \sigma)_{c_{0}}\right\rangle_{\mathrm{CFT}}, \quad \text { with } \quad c_{0}=c_{1}
$$

where the subscript of the pair $(\sigma \sigma)_{c}$ denotes its fusion channel $c$. Similarly, we can represent $n$ qubits as a correlator of $(n+1)$ pairs of Ising anyons $\sigma$ where the last pair compensates the total fermion parity

$$
\left|c_{1}, \ldots, c_{i}, \ldots, c_{n}\right\rangle \rightarrow\left\langle(\sigma \sigma)_{c_{1}} \cdots(\sigma \sigma)_{c_{i}} \cdots(\sigma \sigma)_{c_{n}}(\sigma \sigma)_{c_{0}}\right\rangle_{\mathrm{CFT}}
$$

with $c_{i}= \pm$ being the fermion parity of the $i$-th pair of $\sigma$ fields. This encoding scheme is illustrated in Fig. 1. 


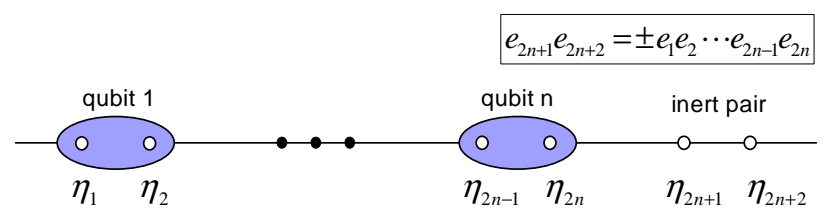

Figure 1. The $n$-qubit encoding scheme: $e_{i}= \pm 1$ is the chiral fermion parity of the $i$-th anyon field $\sigma_{e_{i}}\left(\eta_{i}\right)$ so that $c_{i}=e_{2 i-1} e_{2 i}$, for $1 \leq j \leq n+1$. The sign \pm in the box equation corresponds to the parity of the spinor representation of the braid group

\subsection{Braid matrices: multi-anyon wave-function approach}

The explicit form of the generators of the Ising representation of the braid group $\mathcal{B}_{2 n+2}$ has been conjectured in [8] to coincide with the finite subgroup of the $\pi / 2$-rotations from $\mathrm{SO}(2 n+2)$ (more precisely, with one of the spinor representations of its double cover $\operatorname{Spin}(2 n+2))$. More robust results have been obtained in [9-11]. However, a more natural approach, which is based on the direct computation of the braid matrices by analytic continuation of the multianyon Pfaffian wave functions, using the different operator product expansions of the Ising $\sigma$ fields in the Neveu-Schwarz and Ramond sectors of the Ising model, has been exploited in [12], which allowed to write explicitly the generators $B_{1}^{(2 n+2, \pm)}, \ldots B_{2 n+1}^{(2 n+2, \pm)}$ of the positive/negative parity representations (denoted by \pm in the superscript) of $\mathcal{B}_{2 n+2}$ for arbitrary number $n$ of qubits [12]

$$
\begin{aligned}
B_{2 j-1}^{(2 n+2, \pm)} & =\underbrace{\mathbb{I}_{2} \otimes \cdots \otimes \mathbb{I}_{2}}_{j-1} \otimes\left[\begin{array}{cc}
1 & 0 \\
0 & i
\end{array}\right] \otimes \underbrace{\mathbb{I}_{2} \otimes \cdots \otimes \mathbb{I}_{2}}_{n-j}, \text { for } 1 \leq j \leq n, \\
B_{2 j}^{(2 n+2, \pm)} & =\underbrace{\mathbb{I}_{2} \otimes \cdots \otimes \mathbb{I}_{2}}_{j-1} \otimes \frac{\mathrm{e}^{i \frac{\pi}{4}}}{\sqrt{2}}\left[\begin{array}{cccc}
1 & 0 & 0 & -i \\
0 & 1 & -i & 0 \\
0 & -i & 1 & 0 \\
-i & 0 & 0 & 1
\end{array}\right] \otimes \underbrace{\mathbb{I}_{2} \otimes \cdots \otimes \mathbb{I}_{2}}_{n-j-1},
\end{aligned}
$$

for $n \geq 2$ and $1 \leq j \leq n-1$, as well as

$$
B_{2 n}^{(2 n+2, \pm)}=\underbrace{\mathbb{I}_{2} \otimes \cdots \otimes \mathbb{I}_{2}}_{n-1} \otimes \frac{\mathrm{e}^{i \frac{\pi}{4}}}{\sqrt{2}}\left[\begin{array}{cc}
1 & -i \\
-i & 1
\end{array}\right] .
$$

The last braid generators $B_{2 n+1}^{(2 n+2, \pm)}$ of the $( \pm)$-parity representations of $\mathcal{B}_{2 n+2}$ cannot be written in a similar form for general $n$ because they do not have a tensor product structure. Yet, these diagonal matrices can be determined using Eq. (32) in [6], the results after Eq. (24) in [13] and Proposition 2 in [12]

$$
B_{2 n+1}^{(2 n+2, \pm)}=\frac{\mathrm{e}^{i \frac{\pi}{4}}}{\sqrt{2}}(\mathbb{I}_{2^{n}} \mp i \underbrace{\sigma_{3} \otimes \cdots \otimes \sigma_{3}}_{n}),
$$

where $\sigma_{3}$ is the third Pauli matrix. The above equations (3), (4), (5) and (6) provide the most explicit and compact form of the generators of the two representations of $\mathcal{B}_{2 n+2}$ with opposite fermion parity. Because the Berry connection 
for adiabatic transport of Ising anyons is trivial [7], these braid matrices can be ultimately used to implement topologically protected quantum gates by adiabatic transport in the Ising TQC.

\section{Pauli group for $n$ qubits: quantum correctable errors}

One fundamental structure in any quantum information processing platform is the group of Pauli matrices, or Pauli gates, which are part of the definition of the computational basis. They are important not only because they represent a group of essential quantum operations but also because of the quantum error correction specifics. The point is that there are two independent types of errors which can be considered as deviations of the point, representing the qubit, of the Bloch sphere. Bit-flip $\left(\sigma_{x}\right)$ errors are deviations along the meridians while phase-flip $\left(\sigma_{z}\right)$ errors are deviations along the parallels of the sphere. While it is obvious that arbitrary errors can be decomposed into bit-flip and phase-flip errors, unlike in classical error correction, the errors which could compromise a qubit are continuous quantities. Fortunately, it appears that this continuum of (arbitrary) errors can be corrected by correcting only a discrete subset of those errors: e.g., as in the Shor code [1]. Due to this virtue of the quantum correctability it is sufficient to consider and correct errors which belong to the Pauli group. The $n$-qubit Pauli group is defined as the finite group containing all Pauli matrices $\sigma_{j}$ acting on any of the qubits, including phases of $\pm i$

$$
\mathcal{P}_{n}=\left\{i^{m} \sigma_{\alpha(1)} \otimes \cdots \otimes \sigma_{\alpha(n)} \mid \quad \alpha(j), m \in\{0,1,2,3\}\right\},
$$

(with $\sigma_{0}=\mathbb{I}_{2}$ ). The projective Pauli group is isomorphic to $\mathbb{Z}_{2}^{2 n}$ (see Eq. (17) in [6]), and its center is $\mathbb{Z}_{4}$, so that the order of the $n$-qubit Pauli group (7) is $\left|\mathcal{P}_{n}\right|=2^{2 n+2}$.

\section{1 n-qubit Clifford group: symplectic description}

Because of the fundamental importance of the Pauli group its stabilizer also plays a very important role. By definition the stabilizer of the $n$-qubit Pauli group, known as the $n$-qubit Clifford group, is the group of all unitary $2^{n} \times 2^{n}$ matrices which preserve the Pauli group

$$
\mathcal{C}_{n}=\left\{U \in S U\left(2^{n}\right) \mid U^{*} \cdot \mathcal{P}_{n} \cdot U \subset \mathcal{P}_{n}\right\} .
$$

The fact that the Clifford unitaries commute with the Pauli operators makes them ideal for quantum error correction because they do not introduce new errors while correcting the existing ones.

The Pauli group is naturally a subgroup of the Clifford group (8), i.e., $\mathcal{P}_{n} \subset \mathcal{C}_{n}$. The Clifford group is infinite, however, the projective Clifford group $\left[\mathcal{C}_{n}\right] \equiv \mathcal{C}_{n} / Z$, where $Z$ is its center, is finite. Furthermore, there is an interesting isomorphism [6] between the projective Clifford group $\left[\mathcal{C}_{n}\right]$ factorized by the projective Pauli group $\left[\mathcal{P}_{n}\right] \equiv \mathcal{P}_{n} / \mathbb{Z}_{4}$ and the symplectic group $S p_{2 n}(2)$ (the group of symplectic $2 n \times 2 n$ matrices with elements 0 and 1 )

$$
\left[\mathcal{C}_{n}\right] /\left[\mathcal{P}_{n}\right] \simeq S p_{2 n}(2) .
$$


This isomorphism allows us to compute the order of the projective Clifford group using the known order of the symplectic group $S p_{2 n}(2)$ [14] (see also Appendix A in [6] as well as the order of the projective Pauli group

$$
\left|\mathcal{C}_{n} / Z\right|=2^{n^{2}+2 n} \prod_{j=1}^{n}\left(4^{j}-1\right) .
$$

This result will be important when we try to estimate the computational power of the Ising TQC.

\subsection{Braiding gates as Clifford gates}

A very useful observation in the context of TQC with Ising anyons is that the $n$-qubit Pauli group completely coincides with the monodromy subgroup of the braid group representation $\mathcal{B}_{2 n+2}$

$$
\mathcal{P}_{n} \equiv \text { Image }\left(\mathcal{M}_{2 n+2}\right) .
$$

Then, because the monodromy group is a normal subgroup of the braid group, $\mathcal{M}_{2 n+2} \subset \mathcal{B}_{2 n+2}$, i.e.,

$$
\forall b \in \mathcal{B}_{2 n+2}, \forall m \in \mathcal{M}_{2 n+2}: \quad b^{-1} m b \in \mathcal{M}_{2 n+2},
$$

it follows that all braiding gates are Clifford gates, i.e., the image of the braid group $\mathcal{B}_{2 n+2}$ is a subgroup of the Clifford group for $n$ Ising qubits

$$
\text { Image }\left(\mathcal{B}_{2 n+2}\right) \subset \mathcal{C}_{n} .
$$

To prove this, notice first that $\mathcal{P}_{n} \subset$ Image $\left(\mathcal{M}_{2 n+2}\right)$ because all Pauli gates could be expressed in terms of the squares of the elementary braid generators, which belong to the monodromy group, i.e., for $1 \leq j \leq 2 n+1$, we have for the spinor representations generators $R_{j}^{(n+1, \pm)}$ of $\mathcal{B}_{2 n+2}$ (which have been proven in Proposition 2 in [12] to be equivalent to our representations with generators $B_{j}^{(2 n+2, \pm)}$ derived directly from the wave-function)

$$
\begin{gathered}
\left(R_{2 i-1}^{(n+1,+)}\right)^{2}=\underbrace{\mathbb{I}_{2} \otimes \cdots \otimes \mathbb{I}_{2}}_{i-1} \otimes \sigma_{3} \otimes \underbrace{\mathbb{I}_{2} \otimes \cdots \otimes \mathbb{I}_{2}}_{n-i}, \\
\left(R_{2 i}^{(n+1,+)}\right)^{2}=\underbrace{\mathbb{I}_{2} \otimes \cdots \otimes \mathbb{I}_{2}}_{i-1} \otimes \sigma_{2} \otimes \sigma_{2} \otimes \underbrace{\mathbb{I}_{2} \otimes \cdots \otimes \mathbb{I}_{2}}_{n-i-1} \\
\left(R_{2 n}^{(n+1, \pm)}\right)^{2}=\mp \underbrace{\sigma_{3} \otimes \cdots \otimes \sigma_{3}}_{n-1} \otimes \sigma_{1} \\
\left(R_{2 n+1}^{(n+1, \pm)}\right)^{2}= \pm \underbrace{\sigma_{3} \otimes \cdots \otimes \sigma_{3}}_{n} .
\end{gathered}
$$


Therefore the Pauli gates could be explicitly written in terms of monodromies

$$
\begin{gathered}
\sigma_{2}^{(n)}=i\left(R_{2 n}^{(n+1,+)}\right)^{2}\left(R_{2 n+1}^{(n+1,+)}\right)^{2}, \\
\sigma_{2}^{(n-j)}=i\left(R_{2 n-2 j}^{(n+1,+)}\right)^{2} \sigma_{2}^{(n-j+1)}, \quad 1 \leq j \leq n-1 .
\end{gathered}
$$

On the other hand, as shown in [6], the monodromy generators $A_{i j}$, with $1 \leq$ $i<j \leq 2 n+2$, which can be presented in the form

$$
A_{i j} \equiv U_{i j}^{-1} B_{i}^{2} U_{i j}, \quad \text { where } \quad U_{i j}=\prod_{k=i+1}^{j-1} B_{k},
$$

can be expressed in terms of the Pauli generators [6] due to Eqs. (11), (12), 13) and (14) because

$$
A_{k l}^{ \pm}=-(-i)^{l-k+1}\left(R_{k}^{ \pm}\right)^{2}\left(R_{k+1}^{ \pm}\right)^{2} \cdots\left(R_{l-2}^{ \pm}\right)^{2}\left(R_{l-1}^{ \pm}\right)^{2}
$$

where $1 \leq k<l \leq 2 n+2$ and we omitted the $(n+1)$ in the superscripts. This completes the proof of the statement that all quantum gates which can be implemented by braiding of Ising anyons are actually Clifford gates.

\subsection{Orders of the image of the braid group and the Clifford group}

We saw in the previous subsection that all braiding gates are Clifford gates. Unfortunately, the converse is not true-not all Clifford gates could be implemented by braiding of Ising anyons. To see this let us compare the order of the Clifford group (9) with that of the braid group $\mathcal{B}_{2 n+2}$ in the Ising representation, which is given by $[6,10]$

$$
\left|\operatorname{Image}\left(\mathcal{B}_{2 n+2}\right)\right|=2^{2 n+2}(2 n+2) !, \quad n \geq 2,
$$

and the order for $n=1$ (including the center) is $\left|\operatorname{Image}\left(\mathcal{B}_{4}\right)\right|=96$, see Ref. [15]. Using Eqs. (15) and (9) we compare in Table 1 the orders of the projective braid and Clifford groups for a few qubits. It is obvious that the order

Table 1. Comparison of the orders of the projective image of the braid group $\mathcal{B}_{2 n+2}$ and the projective Clifford group $\mathcal{C}$ for $n$ Ising qubits

\begin{tabular}{l||r|r|r|r|r}
\hline$n$ & 1 & 2 & 3 & 4 & 5 \\
\hline \hline$\left|\mathcal{B}_{2 n+2} / \mathbb{Z}_{4}\right|$ & 24 & 11520 & 2580480 & $\propto 0.9 \times 10^{9}$ & $\propto 0.5 \times 10^{12}$ \\
\hline$\left|\mathcal{P C}_{n}\right|$ & 24 & 11520 & 92897280 & $\propto 1.2 \times 10^{13}$ & $\propto 2.5 \times 10^{19}$ \\
\hline
\end{tabular}

of the Clifford group grows faster with the number of the qubits than the image of the braid group. In Fig. 2 plot the logarithm of the ratio of the order of the projective Clifford group and the order of the image of the braid group, corresponding to $n$ qubits, as a function of the number of qubits $n$. This logarithm still grows quadratically with $n$ which means that the order of the Clifford group grows exponentially faster with $n$ than the order of the image of the braid group. As can be seen from Table 1 the only exceptions are $n=1$ and 2 for which the entire Clifford group could be implemented by braiding [15]. Therefore, it is not possible to realize all Clifford gates for $n \geq 3$ by braiding of Ising anyons. 


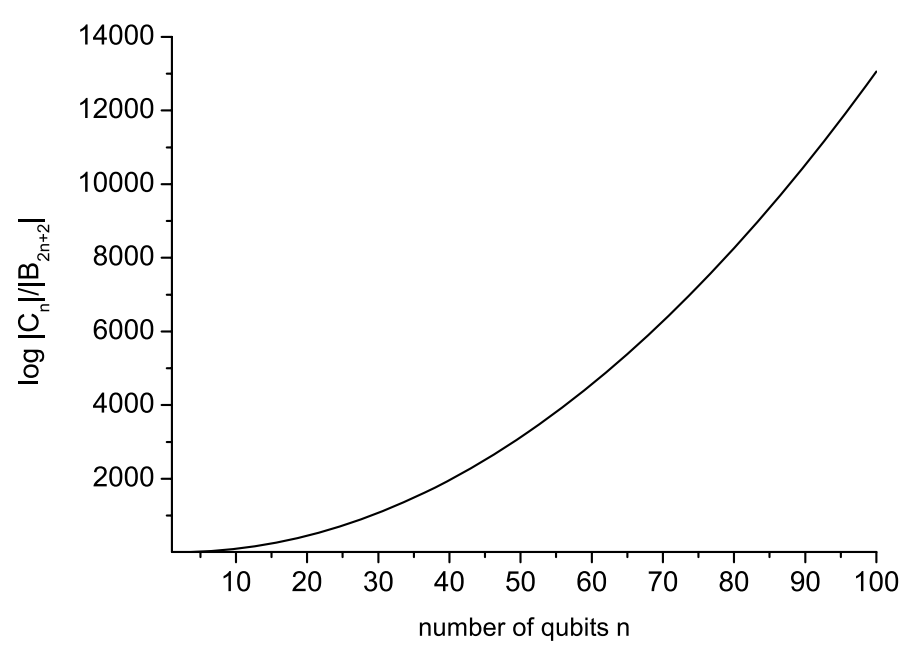

Figure 2. Logarithm of the ratio of the order of the Clifford group and that of the image of the braid group as a function of the number of qubits $n$.

\section{Conclusions}

In this paper we have demonstrated that the $n$-qubit Pauli group for TQC with Ising anyons coincides exactly with the representation of the monodromy subgroup $\mathcal{M}_{2 n+2}$ of the braid group $\mathcal{B}_{2 n+2}$ describing the exchanges of $2 n+2$ Ising anyons. This implies that all braiding gates are actually Clifford gates, which is important for fault-tolerant quantum computation. However, not all Clifford gates are realizable by braiding only. The typically missing Clifford gates from the braid realization are the SWAP gates [6] which simply exchange the quantum states of two qubits in an $n$-qubit quantum register [1]. This is another limitation of the Ising-anyon topological quantum computer, which has already been known [16] to be non-universal for quantum computation since some non-Clifford gates are not realizable by braiding.

\section{Acknowledgments}

We would like to thank Lyudmil Hadjiivanov, Holger Vogts, Sergey Bravyi, Volkher Scholz and Johannes Guetschow for useful discussions. L.S.G. has been supported as a Research Fellow by the Alexander von Humboldt foundation. This work has been partially supported by the BG-NCSR under Contract No. DO 02-257.

\section{References}

[1] M. Nielsen and I. Chuang, Quantum Computation and Quantum Information. Cambridge University Press, 2000. 
[2] A. Kitaev, "Fault-tolerant quantum computation by anyons," Ann. of Phys. (N.Y.) 303 (2003) 2.

[3] S. D. Sarma, M. Freedman, C. Nayak, S. H. Simon, and A. Stern, "Non-Abelian anyons and topological quantum computation," Rev. Mod. Phys. 80 (2008) 1083, arXiv:0707.1889

[4] W. van Dam and M. Howard, "Tight noise thresholds for quantum computation with perfect stabilizer operations," Phys. Rev. Lett. 103 (2009) 170504.

[5] A. Stern, "Anyons and the quantum Hall effect - a pedagogical review," Ann. Phys. 323 (2008) 204-249, arXiv:0711.4697.

[6] A. Ahlbrecht, L. S. Georgiev, and R. F. Werner, "Implementation of Clifford gates in the Ising-anyon topological quantum computer," Phys. Rev. A 79 (2009) 032311, arXiv:0812.2338

[7] N. Read, "Non-abelian adiabatic statistics and Hall viscosity in quantum Hall states and $p_{x}+i p_{y}$ paired superfluids," Phys. Rev. B 79 (2009) 045308, arXiv:0805.2507

[8] C. Nayak and F. Wilczek, " $2 n$ quasihole states realize $2^{n-1}$-dimensional spinor braiding statistics in paired quantum Hall states," Nucl. Phys. B 479 (1996) 529-553, cond-mat/9605145

[9] D. Ivanov, "Non-Abelian statistics of half-quantum vortices in $p$-wave superconductors," Phys. Rev. Lett. 86 (2001) 268-271.

[10] N. Read, "Non-Abelian braid statistics versus projective permutation statistics," $J$. Math. Phys. 44 (2003) 558.

[11] J. Franko, E. C. Rowell, and Z. Wang, "Extraspecial 2-groups and images of braid group representations," J. Knot Theory Ramifications 15 no. 4 (2006) 413, arXiv:math/0503435

[12] L. S. Georgiev, "Ultimate braid-group generators for exchanges of Ising anyons," (2008) arXiv:0812.2334

[13] L. S. Georgiev, "Computational equivalence of the two inequivalent spinor representations of the braid group in the topological quantum computer based on Ising anyons," (2008) arXiv:0812.2337.

[14] R. A. Wilson, The Finite Simple Groups. Springer, Berlin, 2007.

[15] L. S. Georgiev, "Towards a universal set of topologically protected gates for quantum computation with Pfaffian qubits," Nucl. Phys. B 789 (2008) 552-590, hep-th/0611340

[16] M. Freedman, M. Larsen, and Z. Wang, "The two-eigenvalue problem and density of Jones representation of braid groups," (2000) arXiv:math/0103200 